\title{
LA COOPERACIÓN Y EL COOPERATIVISMO COMO RESORTES DEL DESARROLLO
}

\author{
Jaime García Ruiz* \\ Lienny García Pedraza ${ }^{* *}$
}

\begin{abstract}
RESUMEN
La cooperación es tan antigua como la misma sociedad humana. Como vía específica de socialización socialista, potencia el desarrollo socioeconómico territorial y local. Propicia lazos de cooperación del trabajo y del proceso de producción que se dan en la sociedad y que toman cuerpo en las cooperativas. El presente trabajo trata de explicar la importancia de las relaciones de cooperación en el ámbito territorial-local. Para dar respuesta a tal objetivo se emplea la capacitación y los entrenamientos - espacios de educación - a cooperativas y a actores locales vinculados al sector cooperativo. Se combinan con las siguientes técnicas: matriz DAFO, entrevista a presidentes de las juntas directivas de las cooperativas y cuestionarios a socios y actores locales. Los principales resultados están referidos al proceso de cooperación en sus diferentes niveles y se exponen como lecciones aprendidas del proceso.
\end{abstract}

PALABRAS CLAVE: desarrollo local, cooperación, inter cooperación, cooperativismo.

\begin{abstract}
Cooperation is as old as society itself. As a specific way of socialist socialization, it promotes local socioeconomic development. It
\end{abstract}

\footnotetext{
* Jaime García Ruiz (Cuba, 1959). Doctor en Ciencias Económicas por la Universidad de La Habana (1990). Profesor Titular de Economía Política, del Departamento de Filosofia e Historia de la Universidad Central "Marta Abreu" de Las Villas desde 1985. Miembro de la Cátedra honorífica sobre cooperativismo y desarrollo Dr. Víctor M. Figueroa Albelo. Ha publicado libros y artículos en Revistas en Cuba y en el extranjero.

** Lienny García Pedraza. (Cuba 1991). Licenciada en Sociología por la Universidad Central "Marta Abreu" de Las Villas (2014). Profesora asistente del Departamento de Sociología de la Facultad de Ciencias Sociales de la UCLV. Investigadora sobre cooperativismo y desarrollo. Mimbro del Grupo de estudios rurales y agrarios y el Grupo de estudios de trabajo, ambos de la Facultad de Ciencias Sociales. Ha publicado artículos en revistas y libros.
} 
fosters ties of cooperation in the work and production process that take place in society and take shape in cooperatives. The present work tries to explain the importance of cooperative relations at the local level.To respond to this objective, training and training educational spaces - are used for cooperatives and local actors linked to the cooperative sector. They are combined with the following techniques: matrix of weaknesses, threats, strengths and opportunities, interview to presidents of boards of directors and questionnaires to partners and local actors. The main results are related to the cooperation process, in its different levels. They are exposed as lessons learned from the process.

KEYWORDS: local development, cooperation, inter cooperation, cooperativism.

\section{INTRODUCCIÓN}

Lo que ha caracterizado y aún caracteriza a Cuba, es el bajo desarrollo de las fuerzas productivas y la deformación estructural, en buena medida, heredadas del pasado prerrevolucionario. La experiencia histórica entorno a la transición del capitalismo al socialismo ha demostrado que contrario al planteamiento de Carlos Marx y Federico Engels este no es un resultado del alto desarrollo de las fuerzas productivas, sino que ha constituido una alternativa de desarrollo al modelo capitalista llevado a cabo por un país subdesarrollado y periférico inmerso en el sistema capitalista mundial al que nadie escapa. Cuenta con la presencia de un sistema de relaciones sociales de producción y leyes económicas, además de subdesarrolladas, en formación, en germen y, por tanto, inmaduras. Las relaciones sociales de producción nuevas no aparecen de la nada, de un tirón o por obra y gracia de algún ser sobrenatural o deseo de las personas al interpretarlas y perfeccionarlas. Ello también está condicionado, objetivamente, por su madurez.

A Cuba habría que agregarle, además de ser un pequeño país subdesarrollado, con escasos recursos naturales; el hecho de ser generador de un producto interno bruto (PIB) muy pequeño. También con un mercado interno subexplotado, con bajo índice de consumo per cápita y una economía abierta, altamente dependiente del sector externo. Por tales condiciones el proceso de desarrollo económico y social requiere de un largo plazo y su trayectoria no es ni puede ser lineal. Tampoco lo es el crecimiento económico bajo el 
impacto de diversos obstáculos y contradicciones internas y la interdependencia con el mercado globalizado transnacional (incluye los bloques regionales) con sus ciclos recurrentes. $\mathrm{Y}$ « ies que puede encontrarse en la historia un solo ejemplo de un modo de producción nuevo que haya prendido de golpe, sin una larga serie de reveses, equivocaciones y recaídas?» (Lenin, 1961, p. 231).

La reforma del modelo económico persigue los principios que desde 1959 han sido ineludibles para Cuba: la independencia nacional, la equidad y justicia social y la conservación del curso socialista del desarrollo. Los cambios estructurales se implementan en función de desatar el desarrollo de las fuerzas productivas nacionales y la reinserción de la economía cubana en la economía mundial, lo que mantiene el consenso popular.

A lo largo de todos los Lineamientos de la Política Económica y Social del Partido y la Revolución, se expresa que un objetivo estratégico principal es la sustitución de importaciones y la generación de exportaciones. Junto al complejo agroindustrial nacional se sitúa el sector mixto, en el que el turismo ocupa un lugar importante al igual que los servicios científico-técnicos. Ante ello, ¿el Estado debe asumir el papel regulador o supervisor en este proceso?

Una de las funciones de cualquier Estado es la de servir de regulador de las relaciones entre los sujetos económicos. El mayor o menor grado de intervención de este en los asuntos económicos ha dependido del tipo de relaciones sociales imperantes en el país en épocas concretas. A diferencia del capitalismo, en la construcción del socialismo el papel que desempeña el factor subjetivo, así como las posibilidades reales de intervención del Estado en la regulación del movimiento económico y en el establecimiento de las proporciones son definitorios.

Las vías e instrumentos que utiliza, o puede utilizar, el Estado son diversas y cambiables en diferentes momentos. El sistema de contratación económica, el sistema impositivo, la oferta, los ingresos de los consumidores, los precios y el control obrero - la supervisión, el control de inventarios - son algunos de los instrumentos de regulación de la espontaneidad del mercado que debe utilizar el Estado. Estos sistemas deben abarcar todos los tipos de economías o formas productivas sin excepción, ya se trate de la forma estatal, las cooperativas o el sector de los pequeños y medianos propietarios 
privados individuales ${ }^{1}$ o también al interior de cada uno de ellos, entre los productores y los consumidores; etcétera.

Las condiciones del sistema de relaciones sociales de producción que sustenta la economía cubana constituyen una premisa para el presente trabajo. A lo cual debe agregarse que bajo estas condiciones se hace indispensable fortalecer las relaciones de cooperación entre estas formas de propiedad predominantes y al interior de ellas y entre los productores. El cooperativismo está determinado por el sistema de relaciones sociales de producción prevalecientes en la sociedad de que se trate. En su evolución histórica demuestra que ha constituido una alternativa al capitalismo, pero dentro del propio sistema.

En palabras de Carlos Marx el capitalismo, con la cooperación socializa los medios de producción y acelera la elaboración del objeto y establece cadenas entre los productores (Marx, 1973, pp. 278-291). El poder estatal sobre los medios de producción en el socialismo permite la organización de la sociedad en cooperativas. Por tanto, el cooperativismo constituye una forma de socialización que transforma la propiedad privada capitalista en propiedad social. Sin embargo, para lograr una verdadera emancipación social es necesario rebasar los límites del sistema. Esto constituye premisa del presente trabajo, con el cual se pretende explicar la importancia de las relaciones de cooperación en el ámbito local.

Para ello se tienen en cuenta las experiencias del Grupo de estudios rurales y agrarios de la Universidad Central "Marta Abreu" de Las Villas. Los breves resultados expuestos constituyen aprendizajes de los procesos de capacitación y entrenamiento en cooperativas agropecuarias y no agropecuarias del municipio de Placetas, provincia Villa Clara.

El proceso de intervención se estructuró en talleres y se auxilió de técnicas de investigación como la matriz DAFO, las entrevistas a presidentes de las juntas directivas de las cooperativas y la aplicación de cuestionarios. Fueron seleccionadas dos cooperativas no

\footnotetext{
1 "Una cosa es fantasear sobre toda clase de asociación obrera para la construcción del socialismo y otra es aprender en la práctica a construir ese socialismo, de tal modo que cada pequeño campesino (J. García: pequeño productor privado individual) pueda colaborar en esa construcción" (Lenin, 1961, p. 779)
} 
agropecuarias del municipio de Placetas, objeto de los entrenamientos. Diez cooperativas agropecuarias de propio municipio, del tipo CPA y CCS.

Desde los talleres e instrumentos aplicados se fomentó una visión del cooperativismo como movimiento y como forma de propiedad articuladora de las relaciones sociales de producción en el ámbito local.

\section{EL DESARROLLO LOCAL EN LA ACTUALIZACIÓN DEL MODELO SOCIOECONÓMICO CUBANO}

Desde antes del triunfo revolucionario de 1959, científicos y políticos cubanos se han ocupado del desarrollo económico en Cuba. Sin embargo, el desarrollo local es de más reciente introducción (década de 1990) en la academia siendo; tema de investigación como práctica sociopolítica y del gobierno.

El desarrollo local en Cuba adquiere características, carácter y objetivos muy diferentes a los propuestos por las teorías y prácticas neoliberales. De aquí que se deba definir claramente: ¿cuál desarrollo local es el conveniente dadas las características de las localidades (sistema de relaciones sociales de producción) en transición al socialismo en Cuba? Y luego: ¿cuáles son los elementos estructurales y del mecanismo de gestión del modelo económico en actualización vinculados al desarrollo local?

El desarrollo es un proceso universal de carácter objetivo históricamente determinado y de contenido multidimensional, que intervincula las dimensiones económica, social, cultural, ambiental, científico-tecnológica y político-jurídica. Cada una de ellas incluye, a su vez, múltiples categorías, variables e indicadores que se sustentan en un conjunto de principios básicos tales como: la eficiencia, la equidad, la sostenibilidad, la cooperación, la seguridad, la potenciación y la participación (García Ruiz, 2009, p. 14). Debe considerarse que el Informe de Cuba sobre el Desarrollo Humano del año 1996 explicó como «dimensiones del desarrollo» lo que en este análisis se entiende como «principios básicos o valores» del proceso de desarrollo que necesitan nuestras naciones latinoamericanas.

El desarrollo como proceso universal de cambio y transformación acusa su carácter conforme al tipo de relaciones 
sociales de producción prevalecientes, los valores, la ideología e intereses clasistas de la sociedad de que se trate (Engels, 1979, p. 180). Todo ello determina los objetivos, el tipo de mecanismo de realización, las políticas y estrategias de desarrollo, su implementación y los instrumentos jurídicos e institucionales que se empleen.

Por tanto el desarrollo se realiza (materializa) en espacio-tiempo concretos: la sociedad cubana actual, sustentada en un sistema de relaciones sociales de producción heterogéneas $\mathrm{y}$, por demás, contradictorias, donde predominan las relaciones socialistas de producción en transición hacia una nueva cualidad. Entonces, el desarrollo solo puede ser entendido, al explicar las tendencias regulares y al superar las contradicciones de dicho sistema, lo que convierte a este último en el verdadero objeto de investigación (Marx y Engels, 1973, p. XI).

Así, el carácter esencialmente socialista del proyecto social cubano pone en el centro de atención a los seres humanos y su entorno, como gestores directos de su destino y, por otro lado, como beneficiarios directos de los resultados de dicho proyecto.

De lo anterior se desprende que un momento esencial y de partida, en la concepción del desarrollo está, en determinar la estructura, las características y las tendencias (leyes económicas) del sistema socioeconómico dado $\mathrm{y}$, con ello, sus agentes. Deben identificarse las dimensiones $\mathrm{y}$, al interior de ellas, los ejes socioeconómicos fundamentales y los sujetos en su estructuración en clases y grupos sociales. Por ende, la localidad cubana es un sistema heterogéneo compuesto por diferentes tipos de economías, sujetos económicos, grupos y clases sociales altamente contradictorios.

Las contradicciones como fuentes fundamentales de desarrollo tienen un comportamiento dinámico y su solución-superación es parte del dinamismo de los procesos reales. Cuando unas son vencidas otras aparecen o se hacen más visibles. Ellas solo pueden ser analizadas desde esta perspectiva y dinámica. Todo proceso de desarrollo implica cambios inducidos - o no - y, por tanto, la aparición y solución de las contradicciones que este engendra.

En las condiciones de Cuba, además de las contradicciones macroeconómicas generales pueden aparecer otras de carácter local o generado por los cambios que se introducen en el modelo socioeconómico. Como las que se dan entre la acumulación y el 
consumo, entre el consumo realizado a través de los ingresos provenientes del trabajo y los fondos sociales de consumo, entre el desarrollo económico y el desarrollo social, entre la regulación planificada y el mercado, etcétera. Entre estas contradicciones aparecen:

$\checkmark$ los grados de descentralización económico-financiera que se pretende introducir a nivel local y la centralización gubernamental territorial y sectorial;

$\checkmark$ la producción-apropiación de excedentes económicos por los productores directos y los órganos de gobiernos locales y territoriales;

$\checkmark$ la liberación del acceso a los bienes de consumo (eliminación del racionamiento) y el tránsito paulatino al subsidio a los segmentos de la población de más bajos ingresos;

$\checkmark$ la restructuración del empleo (proceso de disponibilidad laboral bajo el principio de idoneidad demostrada) y las ofertas de nuevas alternativas de empleo; y

$\checkmark$ los distintos tipos de economía a nivel local, portadores de intereses económicos diferentes.

Es eminente la necesidad de reconocer lo local como un complejo sistema -totalidad- de relaciones sociales de producción, heterogéneas en sus vínculos e interdependencias. Es ahí, donde se produce la configuración-diferenciación más nítida de las clases y grupos sociales en los territorios con intereses económicos diferentes y contradictorios; los que tendrán que encontrar soluciones a este nivel por los propios actores municipales. Nadie desde fuera podrá hacerlo.

Para ello debe tenerse en cuenta la estructuración sectorial y ramal de los territorios y localidades que se sustenta en la división social del trabajo. La misma es expresión del desarrollo de las fuerzas productivas, la base material del desarrollo. El lado socioeconómico del asunto está vinculado al proceso de socialización socialista de las fuerzas productivas materiales.

El desarrollo del sistema de división social del trabajo, el carácter interdependiente de todos sus componentes hace que la coordinación no puede ser sobre la base de un conocimiento perfecto de las múltiples relaciones como para coordinar de forma directa, consciente y planificadamente el sistema a la perfección. El limitado conocimiento del sistema hace imprescindible la utilización hasta 
cierto punto de las relaciones mercantiles y el mercado. La combinación de ambos es la vía a través de la cual se produce el acercamiento a una coordinación eficiente del sistema. (Hinkelammert, Mora Jiménez, 2014, p. 492)

La división social del trabajo en la localidad y, por tanto, la existencia de diferentes niveles de desarrollo imponen objetivamente el establecimiento de lazos o sistemas de cooperación horizontal e intersectorial a escala local. Ello potenciaría la complementación local entre los distintos sectores y ramas económicas del territorio. Por tanto, una dirección y principio fundamental del desarrollo local está en la potenciación, a este nivel, de la cooperación y de las formas productivas cooperativa y privada individual, así como familiar pequeña y mediana. Las cooperativas deben concebirse en los ámbitos de la producción, del trabajo asociado y de créditos y servicios en la producción agropecuaria, la pequeña industria, la industria local y los servicios, para restablecer los lazos de cooperación e integración locales rotos en la década de 1960. Además, deben aprovechar las capacidades externas, sectoriales y verticales.

La cooperación horizontal en las localidades es concebida al menos desde tres niveles, entre los sectores y ramas de la economía y al interior de estos entre sus eslabones para lograr el máximo posible de complementariedad interna, la cooperación entre los diferentes tipos de economías o formas productivas y al interior de los tipos de economías para elevar lo más posible la socialización socialista a escala local y hacer compatibles los intereses colectivos, individuales, locales y sociales. El cooperativismo en particular-agropecuario y no agropecuario - es una vía específica de socialización socialista que potencia el desarrollo socioeconómico local. Además, las cooperativas y empresas tienen una responsabilidad social empresarial que deberá impulsarse. Los órganos locales de gobierno son los encargados de establecer Sistemas de Cooperación Municipal como métodos de trabajo como catalizadores de la cooperación e integración horizontal. "Es necesario - decía Lenin - organizar políticamente la cooperación de suerte que no solo disfrute en todos los casos de ciertas ventajas, sino que estas sean de índole puramente material (el tipo de interés bancario, etc.)" (Lenin, 1961, pp. 779-780)

Los distintos tipos de cooperativas en Cuba articulan relaciones y prácticas especificas entre sus integrantes, ya sean de 
índole laboral, disciplinaria, de seguridad y de manejo de conflictos internos y gozan de cierto grado de flexibilidad en su régimen económico. Cuentan con una autonomía formal con relación al Estado y están dotadas de un marco legal propio, constituido sobre su propio sistema constitutivo. Poseen vínculos tanto con entidades económicas estatales como con organizaciones políticas, sociales y de masas.

La cooperativización constituye un fenómeno complejo en la vida social en diferentes etapas del desarrollo de la sociedad. $\mathrm{Su}$ papel es manifiesto según la formación económica social existente. El movimiento cooperativo tiene rasgos similares, incluso en diferentes escalones del desenvolvimiento de una misma forma social. Cada formación económica social, determina la composición social y los objetivos inmediatos del movimiento cooperativo ${ }^{2}$ y las posibles vías de su empleo por las fuerzas y movimientos sociales.

En la actualidad se conciben las estrategias y la planificación local para el desarrollo integral de forma consciente, a partir de los recursos disponibles a esta escala y en todas sus dimensiones y principios básicos. Esto debe contrarrestar u oponerse a la espontaneidad que introducirán, objetivamente con más fuerza, los instrumentos monetarios mercantiles y de mercado. Por lo que la contradicción entre la regulación consciente y el mercado regulado con su grado de espontaneidad también tiene que resolverse a este nivel.

El municipio define los pivotes de su desarrollo y sus fuentes de acumulación originarias externas, centralizadas, no centralizadas e internas. Todo este proceso no se limita a cambios en el mecanismo de gestión del desarrollo, sino que pasa por los cambios necesarios en las formas de pensar y actuar de los distintos actores a los diferentes niveles. Esto último se convierte en una de las barreras que deben ser superadas para lograr el objetivo supremo de la política económica y social formulada: «garantizar la continuidad y la irreversibilidad del socialismo, el desarrollo económico del país y la elevación del nivel de vida de la población, conjugado con la

\footnotetext{
${ }^{2}$ Al respecto Vladimir Ilich Lenin en su trabajo Sobre la cooperación afirmaba: "cuando los medios de producción pertenecen a la sociedad, cuando es un hecho el triunfo de la clase del proletariado sobre la burguesía, el régimen de los cooperadores cultos es el régimen socialista." (Lenin, 1961, p. 781)
} 
necesaria formación de valores éticos y políticos de nuestros ciudadanos» (Partido Comunista de Cuba, 2011, p. 10).

Teniendo en cuenta las potencialidades de los municipios, el cooperativismo emerge como motor impulsor del desarrollo y especialmente en las localidades cubanas donde el gobierno tiene la posibilidad de fomentar lazos de cooperación entre los diferentes sectores y actores.

\section{LA INTERCOOPERACIÓN: EXPERIENCIAS EN VILLA CLARA}

La cooperativa constituye un medio institucional directo que propicia la cooperación entre los sujetos que la integran, además de establecer una estructura específica sustentada en sus principios y valores. Sin embargo, la cooperativa nos es la única vía socioeconómico-institucional para establecer vínculos entre los sujetos, producto a que la cooperación no sólo se da a ese nivel. La cooperación se da al interior de las cooperativas - como se expresó anteriormente -, entre las cooperativas y entre el sector cooperativo y otros sectores y actores sociales en los diferentes niveles, aunque esté o no institucionalizada.

No debe ignorarse ninguna de estas relaciones de cooperación, debe comprenderse que, en el caso estudiado, se presta atención a la cooperación que se da al interior de las cooperativas y entre las cooperativas. Por tanto, son ellas los medios institucionales directos para satisfacer determinadas necesidades de los hombres; teniendo como base la forma de propiedad colectiva de la producción, la apropiación, la circulación y el consumo.

Esta interpretación articula varios elementos:

- La cooperación expresa relaciones sociales producción, determinadas por la propiedad colectiva sobre los medios, que van a supeditar las demás relaciones: interpersonales (interrelacionadas y recíproco), grupales (de identidad, de ayuda mutua, asociatividad), sectoriales (al interior del sector y con otros sectores) y locales; que para el caso cubano son predominantes las formas socialistas.

- La cooperación implica objetivos comunes: pueden estar dados por la acción común que permite la realización de varia 
tareas, sobre un mismo objeto y de varios trabajadores al mismo tiempo, una acción auxiliadora que implica la ayuda mutua, cohesión social de las acciones interrelacionadas que posibilita la integración de partes especializas a partir de la correcta distribución de tareas, donde el proceso cooperativo nace al final ( Llombart Bosch, 1985). Ello potenciaría la capacidad productiva del trabajo.

Ante estas concepciones teóricas ¿qué apuntan las experiencias de Villa Clara, desde el municipio de Placetas?

Desde el año 2016, se realiza una serie de talleres de capacitación, entrenamiento y formación en cooperativas agropecuarias - Cooperativas de Créditos y Servicios (CCS) y Cooperativas de Producción Agropecuaria (CPA) - y cooperativas no agropecuarias (CNoA). Estas acciones son resultado del grupo de estudios rurales y agrarios de la Universidad Central "Marta Abreu" de Las Villas, como anteriormente se explicaba. De estas accione se derivan lecciones aprendidas, las cuales se expone a continuación.

En la cooperación al interior de las cooperativas, se reconocen formas simples de cooperación que se expresan al interior de las mismas. El vínculo entre los productores asociados es indispensable para el funcionamiento de dicha organización.

Las cooperativistas no agropecuarias estudiadas muestran interés por la asociación para ejercer una actividad en común, organizada, con valores éticos y patrimonio propio para alcanzar productividad y eficiencia en su gestión, para sus resultados positivos teniendo en cuenta la objetividad en el proceso de su formación.

En las cooperativas agropecuarias la cooperación se expresa en formas de ayuda financiera a los socios, el préstamo de medios de producción y el intercambio de conocimientos entre ellos de manera informal fundamentalmente, pero también por la vía formal - la contratación.

Factores externos también pueden afectar el funcionamiento interno de las cooperativas. Es el caso del desconocimiento de las bases jurídicas que regulan el funcionamiento de las cooperativas por parte de actores locales y cooperativistas.

En cuanto a la cooperación entre las cooperativas, se identifican insuficientes vínculos horizontales entre las mismas. Las acciones se 
dirigen fundamentalmente a ofrecimientos de productor - préstamos y/o ventas de semillas, medios de producción y servicios -, encuentros con otros productores y reparación de entidades en la comunidad. Sin embargo, esto se reconoce por parte de los asociados como un principio indispensable para el desarrollo del movimiento cooperativo.

El sector no agropecuario del municipio de Placetas en la provincia Villa Clara se articula como movimiento a través de los vínculos entre las cooperativas de dicho sector. El grupo provincial de desarrollo local y cooperativismo, compuesto por los presidentes de las CnoA realizan un sistemático seguimiento a la evolución socioeconómica de las mismas. Propicia encuentros de intercambio de experiencias y gestiona la formación de sus socios en los principios cooperativos. Sin embargo es indispensable que las cooperativas promuevan la educación en los valores y principios del cooperativismo, en la misma medida en que crecen el número de socios. Un nuevo ingreso a la cooperativa constituye un reto en materia de educación.

Sin embargo, se identifica un aislamiento entre el sector cooperativo no agropecuario y sector el agropecuario, dado por la desarticulación desde sus fundamentaciones jurídicas, por la no existencia de una norma única que ordene de forma integral el cooperativismo, ni formas organizativo-funcionales que las acompañe desde los proceso de formación-constitución.

La cooperación entre el sector cooperativo y otros sectores es insuficiente. Expresado en los escasos vínculos de las cooperativas con los organismos, instituciones, organizaciones y empresas a nivel local, lo que retarda el desarrollo local y los vínculos de cooperación horizontal. Lo anterior, acompañado de una menuda visibilidad de las cooperativas por parte de actores locales, limita el proceso productivo y de los servicios de las mismas.

Los organismos e instituciones estatales con los que tienen vínculo las cooperativas son fundamentalmente relacionados $\mathrm{y}$ regulados de acurdo con el tipo de producción y servicios. En el caso del sector agropecuario, los principales órganos de relación lo constituyen las empresas e instituciones del Ministerio de la Agricultura y la Asociación Nacional de Agricultores Pequeños. El sector no agropecuario, el vínculo se establece con los ministerios de relación, que en el caso estudiado se refieren al Ministerio de Industria y al Ministerio del Turismo. 
Los vínculos con otros sectores no se expresan a través de relaciones de cooperación, sino que se muestran por medio de relaciones contractuales; ya sea en el proceso de producción (obtención de las materias primas, alquiler de medios de producción) o de la comercialización (redistribución del producto final).

A nivel local existe un desconocimiento de la personalidad jurídica de la empresa cooperativa. Esto expresa una inexperiencia en materia de cooperativismo y la necesidad de la educación cooperativa. Esos factores afecta el propio proceso de contratación de las cooperativas con empresas locales, provinciales y nacionales que conviven en el ámbito local.

\section{CONSIDERACIONES FINALES}

La reforma del modelo económico en marcha persigue los principios que desde 1959 han sido ineludibles para Cuba: la independencia nacional, la equidad y justicia social y la conservación del curso socialista del desarrollo.

A diferencia del capitalismo, en la construcción del socialismo el papel que desempeña el factor subjetivo, así como las posibilidades reales de intervención del Estado en la regulación del movimiento económico y en el establecimiento de las proporciones son definitorios.

La división social del trabajo en la localidad y, por tanto, la existencia de diferentes niveles de desarrollo y tipos de economía imponen objetivamente el establecimiento de lazos o sistemas de cooperación horizontal e intersectorial a escala local.

Una dirección y principio fundamental del desarrollo local está en la potenciación, a este nivel, de la cooperación y de las formas productivas cooperativa y privada individual, así como familiar pequeña y mediana.

La cooperación horizontal en las localidades es concebida al menos desde tres niveles, entre los sectores y ramas de la economía y al interior de estos entre sus eslabones para lograr el máximo posible de complementariedad interna, la cooperación entre los diferentes tipos de economías o formas productivas y al interior de los tipos de economías para elevar lo más posible la socialización socialista a escala local y hacer compatibles los intereses colectivos, individuales, locales y 
sociales. Mientras que, el cooperativismo en particular - agropecuario y no agropecuario - es una vía específica de socialización socialista que potencia el desarrollo socioeconómico local.

\section{REFERENCIAS BIBLIOGRÁFICAS}

CASTRO, R. (2010): «Discurso pronunciado por el General de Ejército Raúl Castro Ruz, Presidente de los Consejos de Estado y de Ministros, en la clausura del Sexto Periodo Ordinario de Sesiones de la Séptima Legislatura de la Asamblea Nacional del Poder Popular en el Palacio de Convenciones, el 18 de diciembre», Granma Internacional Digital, <http://www.granma.ce/espanol/cuba/20diciem-discurso.html>. Centro de Investigaciones de la Economía Mundial (1997): Investigación sobre el desarrollo humano en Cuba de 1996, Editorial Caguayo, La Habana.

ENGELS, F. (1979): Anti-Duhring, Editorial Pueblo y Educación, La Habana.

FIGUEROA ALBELO, V. (1995): «La reforma Económica en Cuba y sus direcciones principales», en VV. AA.: El sector mixto en la economía cubana, Editorial Félix Varela, La Habana.

FIGUEROA ALBELO, V. (2009): Economía Política de la transición al socialismo. Experiencia cubana, Editorial de Ciencias Sociales, La Habana.

GARCÍA RUIZ, J. (2009): «La ciudad sustentable con desarrollo humano sustentable en América Latina: temas de pesquisa», en Desarrollo sustentable e integración para América Latina y el Caribe, Editorial FURG, pp. 11-34.

GUEVARA, E. (2006): Apuntes críticos a la Economía Política, Editorial de Ciencias Sociales, La Habana.

HINKELAMMERT F. y MORA JIMÉNEZ, H. (2014): Hacia una economía de la vida. Editorial Camino, La Habana.

LENIN, V.I. (1960): «El infantilismo "izquierdista” y el espíritu pequeño burgués», en Obras escogidas, t. 2, Editorial Progreso, Moscú, pp. 16-74.

LENIN, V.I. (1961): «Sobre la cooperación», en Obras escogidas, t. 3, Editorial Progreso, Moscú, pp. 778-781.

LENIN, VI. (1961): «Una gran iniciativa», en Obras escogidas, t. III, Editorial Progreso, Moscú, pp. 217-239.

LLOMBART BOSCH, Desamparados (1985): Aproximación a la Sociología de la cooperación. Revesco - Estudios Cooperativos, número 53. Editorial Universidad Complutense, Madrid. Accesado $<<$ http://dilianet.unirioja/descarga/articulo/1148769>>. 
MARX, C. (1979): La ideología alemana, Editora Política, La Habana.

MARX, K. (1973): El Capital, t. I, Editorial de Ciencias Sociales, La Habana.

MARX, K. y F. Engels (1973): «Prólogo», en El Capital, t. I, Editorial de Ciencias Sociales, La Habana, pp. 9-12.

Partido Comunista de Cuba (2011): Lineamientos de la Política Económica y Social del Partido y la Revolución, La Habana. 
